\title{
Prospective Observational Post-marketing Study of Tafluprost $0.0015 \%$ /Timolol $0.5 \%$ Combination Ophthalmic Solution for Glaucoma and Ocular Hypertension: Short-Term Efficacy and Safety
}

Yasutaka Takagi · Hirotaka Osaki · Tomohiro Yamashita · Yasuhiko Kai

Received: July 8, 2016/ Published online: August 4, 2016

(C) The Author(s) 2016. This article is published with open access at Springerlink.com

\section{ABSTRACT}

Introduction: The intraocular pressure (IOP)-lowering effect and safety of tafluprost $0.0015 \% /$ timolol maleate $0.5 \%$ combination ophthalmic solution (Taf-TFC) were investigated in a real-world clinical setting.

Methods: A prospective up to 2-year (more than 1 year) observational study has been initiated to collect data on the IOP, conjunctival hyperemia score, corneal staining score, and adverse events suffered by patients with glaucoma or ocular hypertension treated at 3 months, and up to 2 years (more than 1 year) after initiating treatment with Taf-TFC. The 3-month findings are reported here.

Results: Among 439 patients enrolled at 100 institutions in Japan, most had normal tension

Enhanced content To view enhanced content for this article, go to www.medengine.com/Redeem/F8E4F0602 32A753A.

Y. Takagi

Scientific Information Group, Santen

Pharmaceutical Co., Ltd, Ofukacho Kita-ku, Osaka, Japan

H. Osaki · T. Yamashita $(\bowtie) \cdot$ Y. Kai

Safety Vigilance Group, Santen Pharmaceutical Co., Ltd, Ofukacho Kita-ku, Osaka, Japan

e-mail: tomohiro.yamashita@santen.co.jp glaucoma $(45.3 \%)$ or primary open angle glaucoma (36.0\%). Adverse drug reaction (ADR) occurred in 5.01\%. The important ADRs were conjunctival hyperemia (five patients), blepharitis (four patients), and punctate keratitis (two patients). Serious adverse reactions occurred in two patients (three events). In 410 patients with data both before and after treatment, baseline mean IOP was $17.5 \pm 5.0 \mathrm{mmHg}$, and it was significantly decreased after 1, 2, and 3 months (all $P<0.05$, paired- $t$ test). IOP was significantly reduced in patients switched to Taf-TFC from either prostaglandin or $\beta$-blocker monotherapy. IOP also decreased significantly in patients switched from a prostaglandin/timolol fixed combination, but not in patients switched from concomitant use of a prostaglandin analog and a $\beta$-blocker. The use of Taf-TFC did not worsen the adherence in most patients.

Conclusion: Taf-TFC significantly reduced the IOP in patients with glaucoma or ocular hypertension treated in daily clinical practice with controllable or recoverable ADRs in short period. Taf-TFC was effective regardless of treatment patterns, and particularly, Taf-TFC significantly reduced IOP in cases in which 
requiring the second line therapy as insufficient of monotherapy.

Funding: Santen Pharmaceutical Co., Ltd., Osaka, Japan.

Keywords: Fixed-combination;

Glaucoma; Intraocular pressure; Observational post-marketing study; Ocular hypertension, prostaglandin; Tafluprost; Timolol

\section{INTRODUCTION}

Glaucoma is a chronic disease and still a major cause of blindness. Most patients with glaucoma must use ocular hypotensive drugs continuously throughout their life. However, since glaucoma is relatively asymptomatic in the early stage, the low adherence to ophthalmic solutions for anti-glaucoma was reported [1]. Poor adherence raises the risk of the progression of visual field defects [2] and also the risk of blindness in glaucoma patients [3].

Nowadays, although prostaglandin analogs (PGAs) or $\beta$-blockers are used in the first-line therapy because of their potent ocular hypotensive effects, there are many patients treated with the concomitant use of two or more drugs [4]. The concomitant therapy for glaucoma may cause a decrease in the adherence due to complicated dosing regimens, weaken the hypotensive effects with wash-out effects with a short instillation interval (less than $5 \mathrm{~min}$ ), and cause corneal and/or conjunctival disorders by an increase in the amount and frequency of preservative exposure [5]. Therefore, a fixed combination therapy, when available, should be preferable to two separate instillations of agents [5].

A fixed combination of tafluprost $(0.0015 \%)$ and timolol maleate $(0.5 \%) \quad$ Tapcom $^{\circledR}$ combination ophthalmic solution, Santen
Pharmaceutical Co., Ltd., Osaka, Japan: Taf-TFC) has been developed for the treatment of glaucoma and ocular hypertension $(\mathrm{OH})$. Three Japanese phase III clinical trials conducted in patients with primary open-angle glaucoma (POAG) or $\mathrm{OH}$ have demonstrated that the intraocular pressure (IOP)-lowering effect of Taf-TFC is not inferior to concomitant use of the two active drugs [6] and is superior to either agent alone [6, 7]. Based on these findings, Taf-TFC was approved in Japan in 2014, and it is also marketed in Europe as a preservative-free solution (Tapticom, Santen Oy, Finland). However, typically, the patients in the pivotal clinical studies are limited in terms of the type and severity of glaucoma, associated diseases, and concomitant medications. In addition, treatment was administered under stringent control. To obtain more additional information in real-world setting, a post-marketing study needs to be performed using various treatment regimens in a wider variety of patients to evaluate the efficacy and safety of Taf-TFC in routine clinical practice. Accordingly, Santen is currently conducting a long-term observational study with an up to 2-year (more than 1 year) follow-up period in a large population of more than 1000 patients. This report presents the short-term results obtained with Taf-TFC in various clinical settings based on analysis of the data up to 3 months.

\section{METHODS}

\section{Study Design}

A multicenter, prospective, non-interventional, observational post-marketing study is being conducted in accordance with the requirements of the Japanese regulatory authority (Good Post-Marketing Study Practice, Ministry of 
Health, Labor and Welfare Ordinance No. 171; December 20, 2004). The study commenced in January 2015 and is scheduled to run until September 2018. Since the protocol was reviewed and approved by the Japanese regulatory authority prior to initiation of the study, approval by the ethics committee at each participating institution was not required.

\section{Patients and Registration}

The inclusion criteria for this study are patients with glaucoma or $\mathrm{OH}$ who had not previously been treated with Taf-TFC. A central registration system is used to avoid selection bias. When a patient meets the inclusion criteria, the participating physician registers the patient by facsimile within 2 weeks of prescribing Taf-TFC. Since the Japanese regulatory authority does not require informed consent for post-marketing observational studies, informed consent is not obtained from the patients. The observation period has been set as up to 2 years (more than 1 year) after starting treatment with Taf-TFC. Data are collected over two different periods [0-3 months, and up to 2 years (more than 1 year) after starting treatment], including the demographic profile (data were collected from the physician's report), Taf-TFC regimen, previous and concomitant drugs, IOP, objective findings (conjunctival hyperemia and corneal fluorescein staining), dosing compliance, and adverse events. Patients are classified into the following three groups based on previous and concomitant drugs: "Naïve," no previous treatment or concomitant drugs; "Switched," switched to Taf-TFC monotherapy after treatment with at least one previous drug; "Concomitant," addition of or switching to Taf-TFC combined with other anti-glaucoma medications. A 4-point scale is used to determine the conjunctival hyperemia and corneal staining scores: 0 , none; 1 , mild; 2 , moderate; and 3, severe. If adverse events occur, the physician evaluates the causal relationship with Taf-TFC and events for which a causal relationship to Taf-TFC cannot be excluded are classified as adverse drug reactions (ADRs). Data tabulation and analysis were performed using SAS software (Version 9.2, SAS Institute, Cary, NC, USA), with results being expressed as the mean \pm standard deviation (SD). The starting point of treatment with Taf-TFC was used as the baseline (0 months). The level of statistical significance was set at 5\% (two-sided). The IOP was compared by the paired $t$ test, and the scores for objective findings were compared by Wilcoxon's signed-rank sum test, with the $P$ value then being calculated.

\section{RESULTS}

\section{Demographic Profile}

Data on 439 patients from 100 participating institutions have been analyzed as of December 2015. The demographic and clinical profile of these patients is summarized in Table 1.

In this patient cohort, $45.3 \%$ were male and $54.7 \%$ were female, with a mean age of $67.3 \pm 13.1$ years (range 21-95 years). The diagnosis was POAG (untreated IOP $\geq 21 \mathrm{mmHg}$ ) in $36.0 \%$, normal tension glaucoma (NTG, untreated IOP $<21 \mathrm{mmHg}$ ) in $45.3 \%$, primary angle-closure glaucoma (PACG) in $5.0 \%, \mathrm{OH}$ (untreated $\mathrm{IOP} \geq 21 \mathrm{mmHg}$ without glaucomatous optic neuropathy or glaucomatous visual field loss) in $8.0 \%$, and other types of glaucoma in 5.7\%. Forty-two patients discontinued the study before 3 months, including 9 patients (21.4\%) in 
Table 1 Demographic and clinical profile

\begin{tabular}{|c|c|c|c|c|}
\hline & Total & Naïve & Switched & Concomitant \\
\hline No. of patients analyzed & 439 & 67 & 284 & 88 \\
\hline \multicolumn{5}{|l|}{ Sex } \\
\hline Male & $199(45.3)$ & $30(44.8)$ & $125(44.0)$ & $44(50.0)$ \\
\hline Female & $240(54.7)$ & $37(55.2)$ & $159(56.0)$ & $44(50.0)$ \\
\hline \multicolumn{5}{|l|}{ Age (years) } \\
\hline Mean \pm SD & $67.3 \pm 13.1$ & $65.7 \pm 14.5$ & $67.2 \pm 13.2$ & $68.5 \pm 11.7$ \\
\hline Minimum-maximum & $21.0-95.0$ & $30.0-93.0$ & $21.0-92.0$ & $35.0-95.0$ \\
\hline \multicolumn{5}{|l|}{ Diagnosis } \\
\hline POAG & $158(36.0)$ & $24(15.2)$ & $90(57.0)$ & $44(27.8)$ \\
\hline NTG & $199(45.3)$ & $33(16.6)$ & $141(70.9)$ & $25(12.6)$ \\
\hline PACG & $22(5.0)$ & $2(9.1)$ & $13(59.1)$ & $7(31.8)$ \\
\hline $\mathrm{OH}$ & $35(8.0)$ & $5(14.3)$ & $28(80.0)$ & $2(5.7)$ \\
\hline Other types of glaucoma & $25(5.7)$ & $3(12.0)$ & $12(48.0)$ & $10(40.0)$ \\
\hline \multicolumn{5}{|l|}{ Baseline IOP (mmHg) } \\
\hline Mean \pm SD & $20.0 \pm 6.8$ & $19.1 \pm 7.9$ & $19.5 \pm 5.6$ & $22.5 \pm 8.6$ \\
\hline Min-max & $8.0-52.0$ & $8.0-52.0$ & $10.0-50.0$ & $8.0-52.0$ \\
\hline \multicolumn{5}{|l|}{ Ocular complications } \\
\hline Yes & $149(33.9)$ & $32(47.8)$ & $96(33.8)$ & $21(23.9)$ \\
\hline No & $290(66.1)$ & $35(52.2)$ & $188(66.2)$ & $67(76.1)$ \\
\hline \multicolumn{5}{|l|}{ Systemic complications } \\
\hline Yes & $196(51.0)$ & $27(50.0)$ & $137(54.2)$ & $32(41.6)$ \\
\hline No & $188(49.0)$ & $27(50.0)$ & $116(45.8)$ & $45(58.4)$ \\
\hline Unknown & 55 & 13 & 31 & 11 \\
\hline
\end{tabular}

$I O P$ intraocular pressure, $N T G$ normal tension glaucoma, $O H$ ocular hypertension, $P A C G$ primary angle-closure glaucoma, $P O A G$ primary open-angle glaucoma, $S D$ standard deviation

whom treatment showed insufficient efficacy (4 patients with POAG, 1 patient each with NTG, $\mathrm{OH}$ and angle-closure glaucoma, and 2 patients with other glaucoma type), 14 patients (33.3\%) with adverse events (all events were topical ADRs), 13 patients (31.0\%) who withdrew from study at their own request, and 6 patients (14.3\%) who discontinued for other reasons.
Among the 439 patients, 67 patients (15.3\%) were in the "Naïve" group, 284 patients (64.7\%) were in the "Switched" group, and 88 patients (20.0\%) were in the "Concomitant" group. The characteristics of each group are summarized in Fig. 1. The three groups did not show any large differences of glaucoma type, age, baseline IOP, and baseline mean deviation (MD) values, 


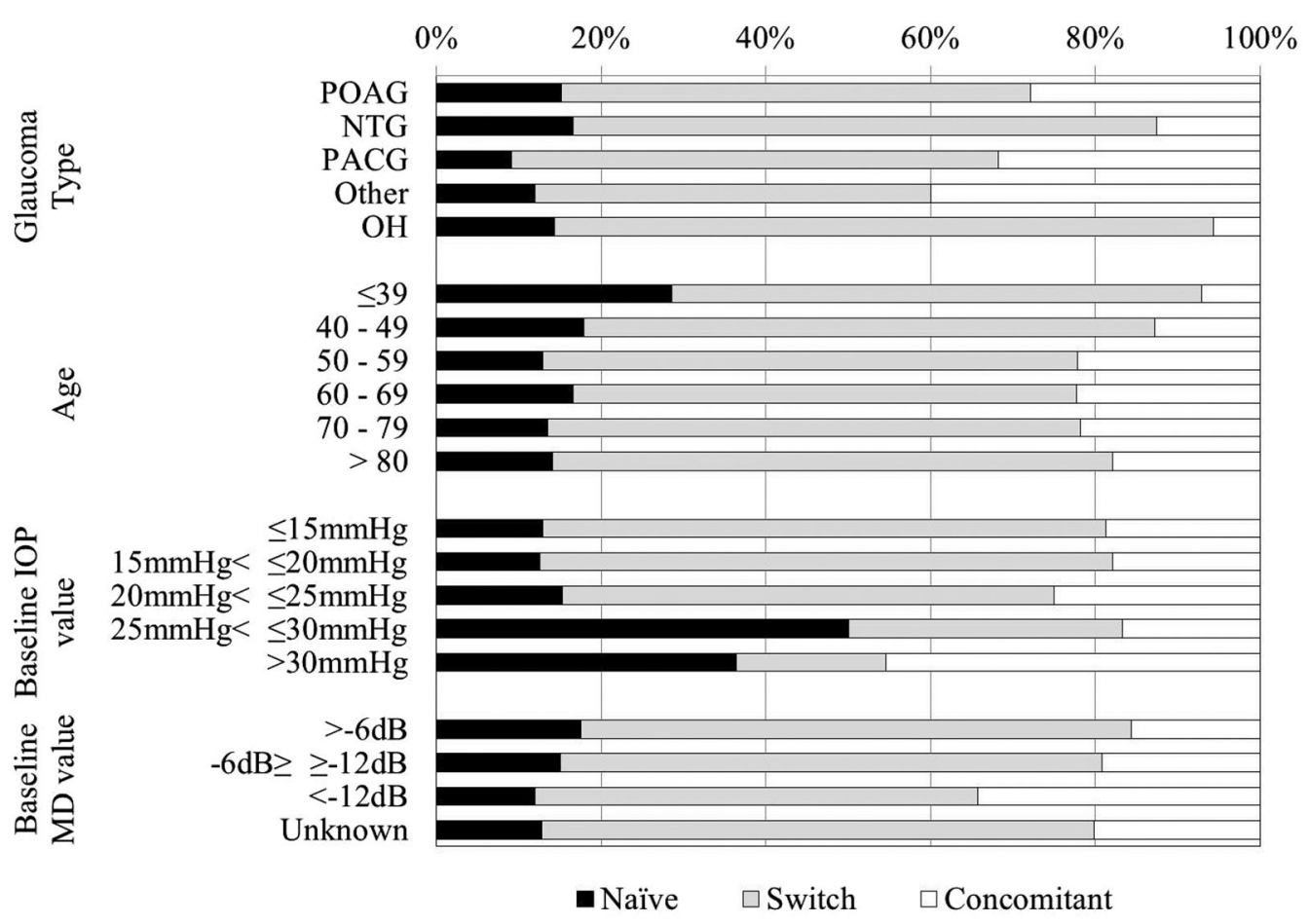

Fig. 1 Background of the three groups. "Naïve" no previous treatment or concomitant drugs; "Switched" switched to Taf-TFC monotherapy after treatment with at least one previous drug; "Concomitant" addition of/switching to Taf-TFC combined with other antiglaucoma medications. IOP intraocular pressure, $M D$

although the "Naïve" group had a higher proportion of patients aged 30-39 years and with a baseline IOP of $25 \mathrm{mmHg}$ or over.

Previous medications varied widely in the "switched" group, including PGA monotherapy ( $n=145,54.5 \%)$, PGA and $\beta$-blocker (timolol, carteolol, etc.) concomitant therapy $(n=34$, $12.8 \%), \quad \beta$-blocker (timolol, carteolol, etc.) monotherapy $\quad(n=28, \quad 10.5 \%), \quad$ and monotherapy with another fixed combination of PGA and timolol (PGA-TFC) $(n=51$, $19.2 \%)$.

\section{Adverse Drug Reactions}

There were 25 adverse events classified as ADRs in 22 patients (5.01\%) (Table 2). ADRs were mean deviation, $N T G$ normal tension glaucoma, $O H$ ocular hypertension, $P A C G$ primary angle-closure glaucoma, $P O A G$ primary open-angle glaucoma, Taf-TFC tafluprost $0.0015 \% /$ timolol maleate $0.5 \%$ combination ophthalmic solution

local (affecting the eye) in 20 events and systemic in 5 events. The major ADRs were conjunctival hyperemia (5 events), blepharitis (4 events), and punctate keratitis ( 2 events). The serious ADR were recognized in 2 patients (3 events); blood pressure increased, asthenic conditions (physical deconditioning), and chest discomfort (one each).

For the case of blood pressure increase, increased blood pressure was observed after 26 days of administration with Taf-TFC who was complicated by hypertension (188/ $108 \mathrm{mmHg}$ at baseline, 207/138 $\mathrm{mmHg}$ at onset). Increased blood pressure was unrecovered $(226 / 142 \mathrm{mmHg})$ at 3 months of administration with continuing Taf-TFC administration. 
Table 2 Adverse drug reactions

No. of patients analyzed

No. of ADRs ${ }^{a}$

No. of patients with ADRs (\%)

System organ class

Preferred term

Events (\%)

Eye disorders

\begin{tabular}{ll} 
Abnormal sensation in eye & $1(0.23)$ \\
Blepharitis & $4(0.91)$ \\
Erythema of eyelid & $1(0.23)$ \\
Eye pain & $1(0.23)$ \\
Eyelid edema & $1(0.23)$ \\
Ocular hyperemia & $1(0.23)$ \\
Photopsia & $1(0.23)$ \\
Punctate keratitis & $2(0.46)$ \\
Vision blurred & $1(0.23)$ \\
Growth of eyelashes & $1(0.23)$ \\
Conjunctival hyperemia & $5(1.14)$ \\
Eye pruritus & $1(0.23)$ \\
Arrhythmia & $1(0.23)$ \\
Palpitations & $1(0.23)$ \\
Asthenia & $1(0.23)$ \\
Chest discomfort & $1(0.23)$ \\
Blood pressure increased & $1(0.23)$ \\
\hline
\end{tabular}

Cardiac disorders

combination

${ }^{a}$ ADR adverse drug reaction (adverse event possibly related to tafluprost $0.0015 \% /$ timolol maleate $0.5 \%$ combination ophthalmic solution)

The asthenic conditions (physical deconditioning) and the chest discomfort were expressed in the same patient and were both expressed after 15 days of administration with Taf-TFC. By the discontinuation of Taf-TFC drug administration, symptoms were recovered.

The mean hyperemia score was $0.2 \pm 0.5$ $(n=386)$ at baseline, $0.2 \pm 0.4 \quad(n=274)$ at 1 month, $0.2 \pm 0.4(n=252)$ at 2 months, and $0.2 \pm 0.4(n=244)$ at 3 months. There was no significant change in the score during the course of the study. The mean corneal staining score was $0.2 \pm 0.4 \quad(n=362)$ at baseline, $0.2 \pm 0.4 \quad(n=256) \quad$ at 1 month, $\quad 0.1 \pm 0.4$ $(n=234)$ at 2 months, and $0.1 \pm 0.4(n=232)$ at 3 months. There were no significant changes of these scores at any time.

\section{IOP-Lowering Effect}

Of the 439 patients, IOP data were analyzed in 410 patients in whom the IOP was measured 
both before and after treatment with Taf-TFC. The treatment patterns of these 410 patients are shown in Fig. 2. Their baseline mean IOP was $17.5 \pm 5.0 \mathrm{mmHg} \quad(n=410)$, and mean IOP changed significantly by $-3.0 \pm 4.2$ at 1 month $(n=282),-3.0 \pm 4.3$ at 2 months $(n=266)$, and $-3.2 \pm 4.3 \mathrm{mmHg}$ at 3 month $(n=243)$ relative to baseline (all $P<0.05$, paired- $t$ test) (Table 3; Fig. 3). IOP was significantly reduced in patients with POAG, NTG, and $\mathrm{OH}$. The IOP-lowering effect of Taf-TFC was not influenced by age or sex.

In the "Naïve" group, the mean IOP was $18.6 \pm 6.1 \mathrm{mmHg}$ at baseline $(n=59)$, and it was significantly reduced at 1,2 , and 3 months

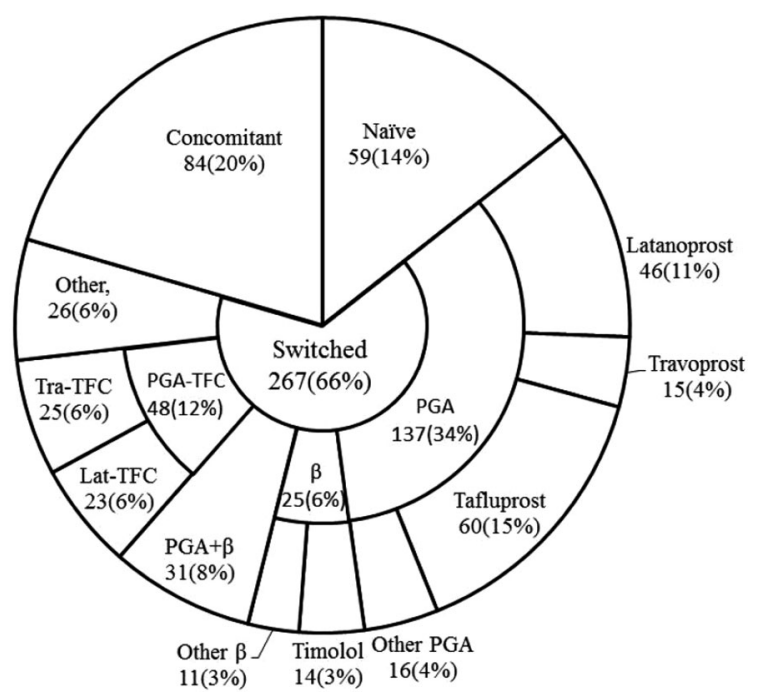

Fig. 2 Treatment patterns in 410 patients. "Naïve" no previous treatment or concomitant drugs; "Switched" switched to Taf-TFC monotherapy after treatment with at least one previous drug; "Concomitant" addition of/ switching to Taf-TFC combined with other antiglaucoma medications. $P G A$ prostaglandin analog, $\beta$ $\beta$-blocker, $P G A-T F C$ fixed combination of a prostaglandin analog and timolol, Lat-TFC fixed combination of latanoprost and timolol, Tra-TFC fixed combination of travoprost and timolol. Lat-TFC latanoprost/timolol combination, PGA prostaglandin analogs, Tra-TFC travoprost/timolol combination
$(-4.9 \pm 3.3,-4.3 \pm 3.0$, and $-5.1 \pm 3.0 \mathrm{mmHg}$, respectively, $P<0.001$, paired $t$-test, Table 3 , Fig. 3). In the "Switched" group, mean IOP was $17.0 \pm 4.2 \mathrm{mmHg}$ at baseline $(n=267)$, and it was also significantly reduced at 1,2 , and 3 months $\quad(-2.1 \pm 2.7, \quad-2.5 \pm 3.1, \quad$ and $-2.4 \pm 3.1 \mathrm{mmHg}$, respectively, $P<0.001$, paired t-test, Table 3, Fig. 3). In the "Concomitant" group, mean IOP was $18.2 \pm 6.4 \mathrm{mmHg}$ at baseline $(n=84)$, and it likewise showed a significant decrease at 1,2 , and 3 months $(-4.5 \pm 6.9,-3.9 \pm 6.9$, and $-4.6 \pm 6.9 \mathrm{mmHg}$ respectively, $P<0.001$, paired t-test, Table 3, Fig. 3).

In patients switched from PGA monotherapy to Taf-TFC monotherapy, mean IOP was $17.4 \pm 4.0 \mathrm{mmHg}$ at baseline $(n=137)$, and it declined significantly at 1,2 , and 3 months after switching $(-2.8 \pm 2.1,-3.1 \pm 3.2$, and $-2.9 \pm 3.3 \mathrm{mmHg}$, respectively, $P<0.001$, paired t-test, Table 4, Fig. 4). The IOP reduction was similar in patients switched from latanoprost, travoprost or tafluprost to Taf-TFC monotherapy (Fig. 5). Among patients switched from PGA monotherapy, IOP reduction $\geq 2 \mathrm{mmHg}$ was defined as "improved," change of the IOP within $2 \mathrm{mmHg}$ was defined as "unchanged," and an increase in IOP by $\geq 2 \mathrm{mmHg}$ was defined as "worse." As a result, $64.3 \%$ of patients were "improved," 31.0\% were "unchanged," and $4.8 \%$ were "worse" (Fig. 6). At 3 months, improvement was noted in 17 patients (70.8\%) switched from latanoprost $(n=24), 24$ patients $(60.0 \%)$ switched from tafluprost $(n=40)$, and 5 patients $(55.6 \%)$ switched from travoprost $(n=9)$.

In patients switched from $\beta$-blocker (timolol, carteolol, etc.) monotherapy to Taf-TFC monotherapy, mean IOP was $16.7 \pm 3.7 \mathrm{mmHg}$ at baseline $(n=25)$, and it was significantly 
Table 3 IOP stratified by treatment pattern

\begin{tabular}{|c|c|c|c|c|}
\hline Treatment pattern & Baseline & 1 month & 2 months & 3 months \\
\hline \multicolumn{5}{|l|}{ All } \\
\hline $\mathrm{IOP}(\mathrm{mmHg})$ & $17.5 \pm 5.0$ & $14.6 \pm 4.1$ & $14.5 \pm 3.7$ & $14.4 \pm 3.6$ \\
\hline IOP change $(\mathrm{mmHg})$ & - & $-3.0 \pm 4.2$ & $-3.0 \pm 4.3$ & $-3.2 \pm 4.3$ \\
\hline No. of patients & 410 & 282 & 266 & 243 \\
\hline$P$ value & - & $<0.001$ & $<0.001$ & $<0.001$ \\
\hline \multicolumn{5}{|l|}{ Naïve $^{a}$} \\
\hline IOP (mmHg) & $18.6 \pm 6.1$ & $12.8 \pm 3.3$ & $13.8 \pm 3.8$ & $13.9 \pm 3.2$ \\
\hline IOP change $(\mathrm{mmHg})$ & & $-4.9 \pm 3.3$ & $-4.3 \pm 3.0$ & $-5.1 \pm 3.0$ \\
\hline No. of patients & 59 & 45 & 31 & 30 \\
\hline$P$ value & - & $<0.001$ & $<0.001$ & $<0.001$ \\
\hline \multicolumn{5}{|l|}{ Switched ${ }^{\mathrm{b}}$} \\
\hline IOP (mmHg) & $17.0 \pm 4.2$ & $15.0 \pm 4.2$ & $14.4 \pm 3.5$ & $14.5 \pm 3.7$ \\
\hline IOP change $(\mathrm{mmHg})$ & - & $-2.1 \pm 2.7$ & $-2.5 \pm 3.1$ & $-2.4 \pm 3.1$ \\
\hline No. of patients & 267 & 181 & 174 & 162 \\
\hline$P$ value & - & $<0.001$ & $<0.001$ & $<0.001$ \\
\hline \multicolumn{5}{|l|}{ Concomitant $^{\mathrm{c}}$} \\
\hline IOP (mmHg) & $18.2 \pm 6.4$ & $14.6 \pm 4.1$ & $14.9 \pm 4.1$ & $14.2 \pm 3.7$ \\
\hline IOP change $(\mathrm{mmHg})$ & - & $-4.5 \pm 6.9$ & $-3.9 \pm 6.9$ & $-4.6 \pm 6.9$ \\
\hline No. of patients & 84 & 56 & 61 & 51 \\
\hline$P$ value & - & $<0.001$ & $<0.001$ & $<0.001$ \\
\hline
\end{tabular}

IOP intraocular pressure, Taf-TFC tafluprost $0.0015 \% /$ timolol maleate $0.5 \%$ combination ophthalmic solution

a "Naïve," no previous treatment or concomitant drugs

b "Switched," switched to Taf-TFC monotherapy after treatment with at least one previous drug

c "Concomitant," addition of/switching to Taf-TFC combined with other anti-glaucoma medications

reduced at 1, 2, and 3 months after switching to Taf-TFC $\quad(-2.5 \pm 1.9, \quad-2.6 \pm 2.5, \quad$ and $-4.1 \pm 2.1 \mathrm{mmHg}, \quad$ respectively, $P<0.05$, paired $t$-test) (Table 4; Fig. 4).

In patients switched from PGA and $\beta$-blocker (timolol, carteolol, etc.) combined therapy to Taf-TFC monotherapy, mean IOP was $15.1 \pm 2.9 \mathrm{mmHg}$ at baseline $(n=31)$ and did not change significantly up to 3 months of Taf-TFC treatment (paired $t$-test, Table 4, Fig. 4).
On the other hand, in patients with switched from other PGA-TFC to Taf-TFC monotherapy, mean IOP was $18.0 \pm 4.9 \mathrm{mmHg}$ at baseline, and it was significantly reduced at 1,2 , and 3 months after switching to Taf-TFC $(-1.6 \pm 2.2,-1.9 \pm 2.3$, and $-1.3 \pm 3.1 \mathrm{mmHg}$, respectively, $P<0.05$, paired $t$-test, Table 4 , Fig. 4). The decrease in IOP with Taf-TFC monotherapy was similar in patients switched from the latanoprost/timolol combination 


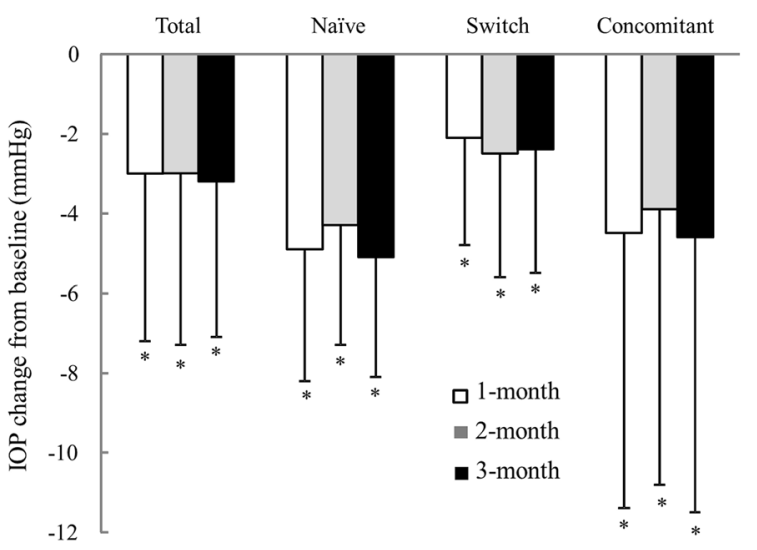

Fig. 3 IOP changes from baseline in 410 patients. "Naïve," no previous treatment or concomitant drugs; "Switched," switched to Taf-TFC monotherapy after treatment with at least one previous drug; "Concomitant," addition of/ switching to Taf-TFC combined with other anti-glaucoma medications. IOP intraocular pressure, Taf-TFC tafluprost $0.0015 \% /$ timolol maleate $0.5 \%$ combination ophthalmic solution

(Lat-TFC) or travoprost/timolol combination (Tra-TFC) drug, but a significant reduction was not observed in the former patients at 1 month and in the latter patients at 3 months (Table 4 , Fig. 7). At 3 months, six patients (46.2\%) switched from Lat-TFC $(n=13)$ and six patients (50.0\%) switched from Tra-TFC $(n=12)$ were classified as "improved" (Fig. 8).

Among the 439 patients, 240 patients instilled Taf-TFC in the morning (before 12:00) and 33 patients used it in the evening (after 16:00). The baseline mean IOP was $17.2 \pm 5.0 \mathrm{mmHg}(n=240)$ in patients with morning dosing and $17.3 \pm 4.3 \mathrm{mmHg}(n=33)$ in patients with evening dosing. With morning administration of Taf-TFC, IOP was reduced significantly to $14.2-14.7 \mathrm{mmHg} \quad(2.7-3.1$ $\mathrm{mmHg}, 12.8-15.3 \%$ decrease), while evening dosing of Taf-TFC significantly reduced the IOP to $14.1-14.5 \mathrm{mmHg} \quad(2.4-3.9 \mathrm{mmHg}$, 12.3-20.4\% decrease) (Fig. 9).

\section{Adherence}

Patient-reported adherence to Taf-TFC therapy was generally good. As a comparison to prior medication, we have analyzed data on 316 patients, whose data of both prior medication and self-reported adherence were available. Among these 316 patients, only 6 patients (1.9\%) answered that compliance with medication was worse at 3 months than with prior medication. The percentage of patients who answered that compliance was improved was similar in the "switched" group and the "concomitant" group $(20.4 \%$ and 19.5\%, respectively). The number of medications resulted in patient adherence to medication being improved (Fig. 10).

\section{DISCUSSION}

This is the first report on post-marketing surveillance of Taf-TFC (Tapcom combination ophthalmic solution) and the first clinical data for Taf-TFC obtained in the real clinical setting since its launch. In brief, Taf-TFC reduced IOP and was well tolerated over a short period.

The majority of patients in this study had NTG or POAG, accounting for more than $80 \%$ of the subjects combined. The Tajimi Study, an epidemiological study conducted in Japan by the Japan Glaucoma Society, found that $72 \%$ of glaucoma patients had NTG $[8,9]$. Almost half of the glaucoma patients in our study had NTG, also showing that NTG is common among Japanese glaucoma patients. Besides patients with NTG, POAG, and OH, our study included patients with PACG, who have not been thoroughly investigated in previous clinical trials. Taf-TFC significantly reduced IOP in patients with NTG, POAG, and $\mathrm{OH}$, and it also 
Table 4 Intraocular pressure stratified by previous medications

\begin{tabular}{|c|c|c|c|c|}
\hline Switched from & Baseline & 1 month & 2 months & 3 months \\
\hline \multicolumn{5}{|l|}{ PGA monotherapy } \\
\hline $\mathrm{IOP}(\mathrm{mmHg})$ & $17.4 \pm 4.0$ & $14.4 \pm 3.3$ & $14.1 \pm 3.0$ & $14.2 \pm 3.3$ \\
\hline IOP change $(\mathrm{mmHg})$ & - & $-2.8 \pm 2.1$ & $-3.1 \pm 3.2$ & $-2.9 \pm 3.3$ \\
\hline No. of patients & 137 & 95 & 85 & 84 \\
\hline$P$ value & - & $<0.001$ & $<0.001$ & $<0.001$ \\
\hline \multicolumn{5}{|l|}{ Latanoprost } \\
\hline IOP $(\mathrm{mmHg})$ & $17.4 \pm 4.6$ & $14.1 \pm 3.1$ & $13.6 \pm 2.7$ & $14.1 \pm 3.2$ \\
\hline IOP change $(\mathrm{mmHg})$ & - & $-3.2 \pm 2.4$ & $-3.8 \pm 4.3$ & $-3.3 \pm 2.7$ \\
\hline No. of patients & 46 & 36 & 26 & 24 \\
\hline$P$ value & - & $<0.001$ & $<0.001$ & $<0.001$ \\
\hline \multicolumn{5}{|l|}{ Travoprost } \\
\hline IOP $(\mathrm{mmHg})$ & $16.6 \pm 3.4$ & $14.8 \pm 3.8$ & $13.9 \pm 3.6$ & $13.4 \pm 3.5$ \\
\hline IOP change $(\mathrm{mmHg})$ & & $-2.1 \pm 1.6$ & $-1.9 \pm 1.6$ & $-2.8 \pm 2.8$ \\
\hline No. of patients & 15 & 10 & 9 & 9 \\
\hline$P$ value & - & 0.003 & 0.008 & 0.016 \\
\hline \multicolumn{5}{|l|}{ Tafluprost } \\
\hline IOP $(\mathrm{mmHg})$ & $16.7 \pm 3.8$ & $14.3 \pm 3.5$ & $13.6 \pm 2.8$ & $13.9 \pm 3.5$ \\
\hline IOP change $(\mathrm{mmHg})$ & & $-2.7 \pm 2.0$ & $-3.1 \pm 2.9$ & $-2.5 \pm 4.0$ \\
\hline No. of patients & 60 & 43 & 38 & 40 \\
\hline$P$ value & - & $<0.001$ & $<0.001$ & $<0.001$ \\
\hline \multicolumn{5}{|l|}{$\beta$-blocker monotherapy } \\
\hline IOP $(\mathrm{mmHg})$ & $16.7 \pm 3.7$ & $14.7 \pm 3.1$ & $13.3 \pm 3.0$ & $13.4 \pm 3.1$ \\
\hline IOP change (mmHg) & - & $-2.5 \pm 1.9$ & $-2.6 \pm 2.5$ & $-4.1 \pm 2.1$ \\
\hline No. of patients & 25 & 18 & 14 & 17 \\
\hline$P$ value & - & $<0.001$ & 0.002 & $<0.001$ \\
\hline \multicolumn{5}{|l|}{ PGA-TFC monotherapy } \\
\hline IOP $(\mathrm{mmHg})$ & $18.0 \pm 4.9$ & $16.6 \pm 5.0$ & $16.4 \pm 4.1$ & $17.0 \pm 5.0$ \\
\hline IOP change $(\mathrm{mmHg})$ & - & $-1.6 \pm 2.2$ & $-1.9 \pm 2.3$ & $-1.3 \pm 3.1$ \\
\hline No. of patients & 48 & 28 & 36 & 25 \\
\hline$P$ value & - & $<0.001$ & $<0.001$ & 0.04 \\
\hline \multicolumn{5}{|l|}{ Lat-TFC } \\
\hline $\mathrm{IOP}(\mathrm{mmHg})$ & $18.0 \pm 5.7$ & $17.4 \pm 6.3$ & $16.9 \pm 5.0$ & $15.2 \pm 4.9$ \\
\hline IOP change $(\mathrm{mmHg})$ & & $-1.3 \pm 2.5$ & $-1.9 \pm 2.6$ & $-1.5 \pm 2.5$ \\
\hline
\end{tabular}


Table 4 continued

\begin{tabular}{|c|c|c|c|c|}
\hline Switched from & Baseline & 1 month & 2 months & 3 months \\
\hline No. of patients & 23 & 13 & 16 & 13 \\
\hline$P$ value & - & 0.08 & 0.01 & 0.049 \\
\hline \multicolumn{5}{|l|}{ Tra-TFC } \\
\hline IOP $(\mathrm{mmHg})$ & $18.1 \pm 4.1$ & $15.9 \pm 3.7$ & $16.0 \pm 3.4$ & $18.9 \pm 4.7$ \\
\hline IOP change $(\mathrm{mmHg})$ & & $-1.8 \pm 1.9$ & $-1.9 \pm 2.2$ & $-1.1 \pm 3.7$ \\
\hline No. of patients & 25 & 15 & 20 & 12 \\
\hline$P$ value & - & 0.002 & 0.001 & 0.315 \\
\hline \multicolumn{5}{|c|}{ Concomitant therapy (PGA, $\beta$-blocker) } \\
\hline IOP (mmHg) & $15.1 \pm 2.9$ & $14.9 \pm 3.9$ & $14.5 \pm 4.0$ & $14.4 \pm 3.6$ \\
\hline IOP change $(\mathrm{mmHg})$ & - & $-0.4 \pm 2.7$ & $-0.7 \pm 3.5$ & $-0.4 \pm 2.1$ \\
\hline No. of patients & 31 & 22 & 22 & 20 \\
\hline$P$ value & - & 0.458 & 0.339 & 0.408 \\
\hline
\end{tabular}

IOP intraocular pressure, Lat-TFC latanoprost/timolol combination, $P G A$ prostaglandin analogs, Tra-TFC travoprost/timolol combination

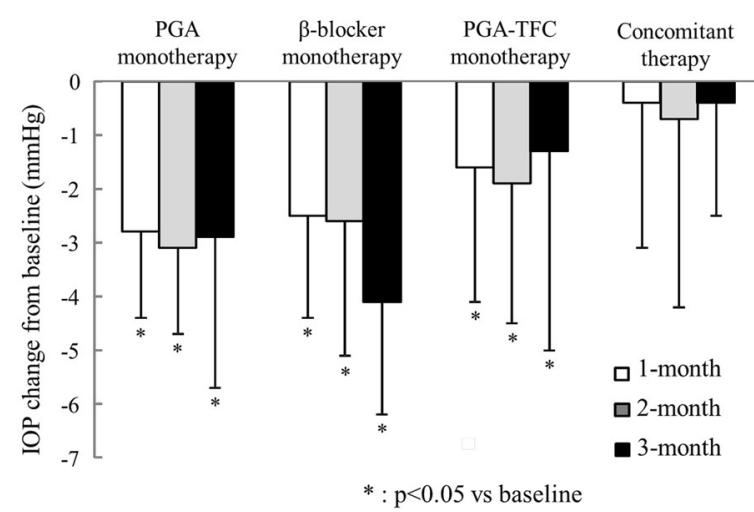

Fig. 4 Intraocular pressure changes from baseline in patients stratified by switched medications. “ $P G A$ ” prostaglandin analog (latanoprost, travoprost, tafluprost, bimatoprost, or unoprostone); " $\beta$-blocker" $\beta$-adrenergic receptor antagonist (timolol or carteolol); " $P G A-T F C$ " fixed combination of a prostaglandin analog and timolol (latanoprost/timolol or travoprost/timolol); "concomitant" concomitant use of a PGA and a $\beta$-blocker. IOP intraocular pressure, $P G A-T F C$ prostaglandin analogs and timolol

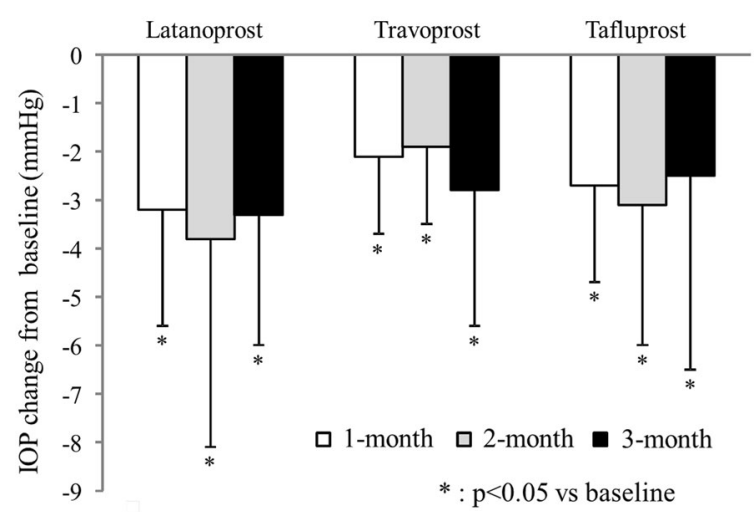

Fig. 5 Intraocular pressure changes from baseline in patients switched from prostaglandin analog monotherapy. $I O P$ intraocular pressure

tended to reduce IOP in PACG patients. These results suggest that Taf-TFC is effective for treating a wide variety of types of glaucoma in actual clinical practice. 


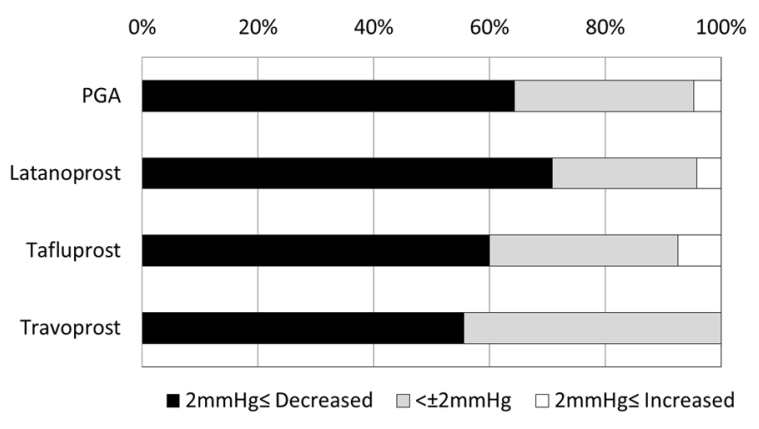

Fig. 6 Efficacy of tafluprost/timolol fixed combination in patient switched from each prostaglandin analog at 3 months. $P G A$ prostaglandin analogs

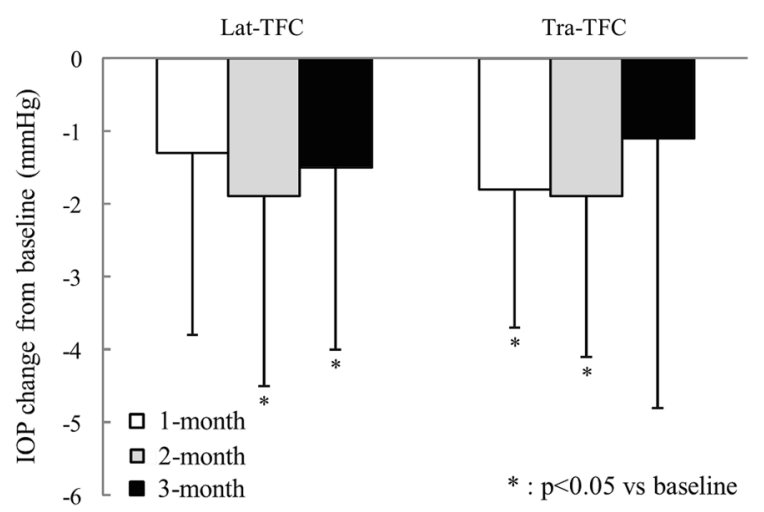

Fig. 7 Intraocular pressure changes from baseline in patients switched from a prostaglandin analog/timolol combination. IOP intraocular pressure, Lat-TFC latanoprost/timolol combination

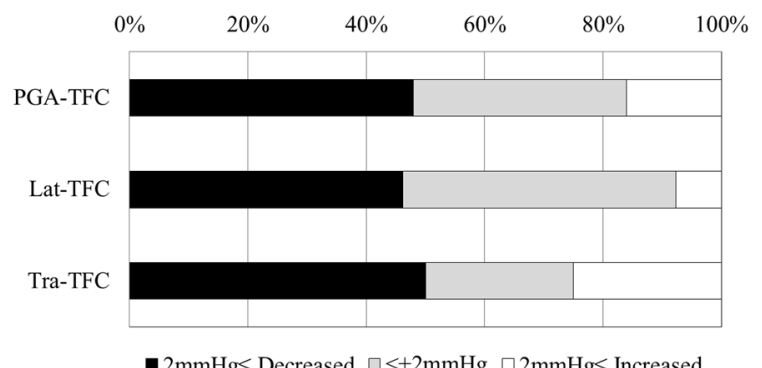

Fig. 8 Efficacy of tafluprost/timolol fixed combination in patients switched from each prostaglandin analog/timolol combination at 3 months. Lat-TFC latanoprost/timolol combination, $P G A-T F C$ prostaglandin analogs and timolol, Tra-TFC travoprost/timolol combination

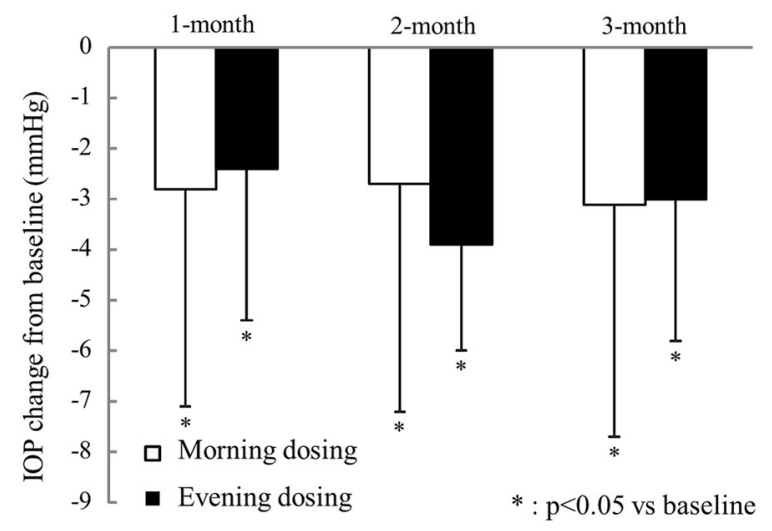

Fig. 9 IOP changes with morning dosing (before noon) or evening dosing (after $4 \mathrm{pm}$ ). IOP intraocular pressure

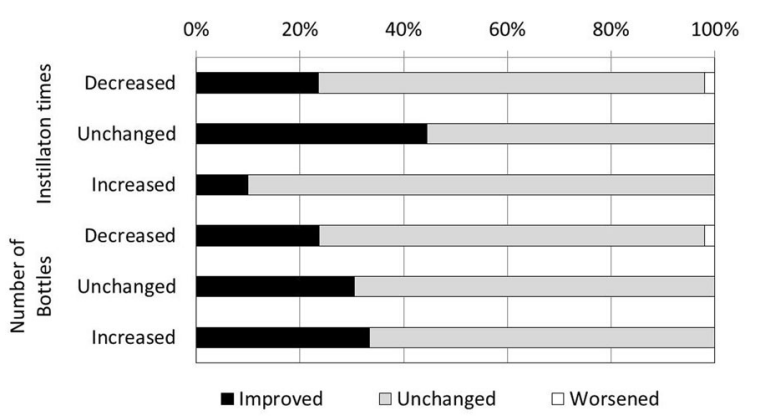

Fig. 10 Adherence to tafluprost/timolol fixed combination compared with previous medication

In this study, the incidence of ADR was $5.01 \%$, which was lower than in previous interventional clinical studies $[6,7]$. This may have been related to differences in the extent of clinical monitoring for adverse events between an interventional clinical study and daily clinical practice. In this study, serious ADRs were observed in two patients. While uncommon, serious ADRs must be considered when starting Taf-TFC. The incidence of ADRs in this study was also lower than that reported in a post-marketing study of Lat-TFC in Japan (9.9\%) [10]. Since this interim report only covers a 3-month period, the incidence of 
ADRs might increase later, and thus the overall safety of Taf-TFC should be concluded after this study have been completed.

In this study, Taf-TFC significantly reduced the IOP. In the Japanese phase III clinical study, the efficacy of Taf-TFC was significantly greater than that of tafluprost monotherapy [6] or timolol monotherapy [7], and was non-inferior to that of tafluprost combined with timolol [6]. In this study, the reduction of IOP in patients switched from PGA monotherapy or $\beta$-blocker monotherapy was similar to that seen in the phase III studies, confirming superior efficacy of Taf-TFC over PGA or $\beta$-blocker monotherapy in this real-world study. Previous studies have shown that some patients exhibit a poor response to various PGAs [11-13]. Some reports indicate that poor responders to one PGA might respond to another PGA [13-15]. In this study, there were no obvious differences of the IOP reduction between patients switched from tafluprost, latanoprost, or travoprost, indicating that Taf-TFC reduces the IOP in patients previously treated with any PGA. Switching to Taf-TFC from another PGA-TFC (Lat-TFC or Tra-TFC) also significantly reduced the IOP, but switching to Taf-TFC from combined PGA and $\beta$-blocker therapy did not significantly reduce and maintain the IOP. In general, although fixed combinations usually have clinical equivalence to unfixed combinations, slight differences in IOP-lowering efficacy may be seen in some cases. It is sometimes assumed that the IOP-lowering effect of fixed combination drugs is weaker than concomitant use of the individual active ingredients, because the daily dose of one active ingredient is decreased. The difference of the IOP reduction noted in patients switched from other PGA-TFCs or concomitant PGA and $\beta$-blocker therapy suggests that the IOP-lowering effect of other
PGA-TFC therapy is weaker than that of concomitant PGA and $\beta$-blocker therapy. However, once daily Taf-TFC was reported to show non-inferiority to concomitant use of tafluprost (once daily) and timolol (twice daily) [6]. Thus, this study confirmed the phase III clinical results in the daily clinical setting. The formulation of Taf-TFC ophthalmic solution was designed to obtain similar penetration of timolol into the anterior chamber as with once daily timolol gel-forming solution, and this was achieved by adjusting the $\mathrm{pH}$ [16]. Penetration of timolol into the anterior chamber is $\mathrm{pH}$ dependent and increases with an increase in $\mathrm{pH}$, because timolol is a basic compound. Indeed, penetration of timolol into the anterior chamber was greater after instillation of Taf-TFC than after a single dose of timolol, and almost equal to that obtained with timolol gel-forming solution [16]. This increased penetration of timolol into the anterior chamber with Taf-TFC may explain why its IOP-lowering effect is equal to that of concomitant PGA and $\beta$-blocker therapy, which may not be the case for all PGA-TFCs. There was also a significant IOP-lowering effect when patients were switched from other PGA-TFCs, with a mean IOP reduction of about $1.5 \mathrm{mmHg}$. Clinically, reduction of the IOP by $1 \mathrm{mmHg}$ is considered to be significant, because the Early Manifest Glaucoma Trial (ClinicalTrials.gov identifier, NCT00000132) revealed an estimated $10 \%$ decrease in the risk of progression for each $1 \mathrm{~mm}$ of mercury reduction in IOP [17].

The question about dosing time of PGA-TFCs is under debate. Timolol is recommended to be used in the morning, because the IOP-lowering effect of timolol maleate is weaker with dosing at night compared to that with daytime dosing [18]. 
On the other hand, the IOP-lowering effect of PGAs does not change throughout the day [18] and most physicians prefer to dose them in the evening. In addition, the theory on which dosing time is better for PGA-TFCs is still controversial. Takmaz reported that while both morning and evening dosing of Lat-TFC were effective for lowering the IOP, evening dosing was more effective than morning dosing in terms of IOP fluctuation [19]. In contrast, there were no differences reported between morning and evening dosing of Lat-TFC [20] and Tra-TFC [21]. Although no large-scale comparison on the effect of the dosing time is available, the results of this study indicate that both morning and evening dosing for Taf-FDC can be utilized.

Adherence to glaucoma medication is an unsolved problem. In principle, treatment of glaucoma should start with monotherapy using a first-line drug followed by addition of a second-line drug or switching to a fixed combination according to many glaucoma guidelines (Japan Glaucoma Society, European Glaucoma Society, etc.). Concomitant use of two or more ophthalmic solutions may reduce adherence compared to monotherapy [22], and the physician must evaluate whether a patient is likely to adhere to complicated treatment regimen. Prescription of a fixed combination is an alternative to two different drugs. In this study, adherence to Taf-TFC was generally good, and only six patients (1.9\%) reported that compliance was worse than to previous medication. Djafari concluded that the use of fewer medications may improve adherence [22]. In this study, the compliance rate showed the greatest improvement in patients who decreased the number of separate medications, but the improvement in compliance was not prominent.
Fixed combination drugs are not the first choice for treating glaucoma except for patients with a high risk for progression or for patients with a severe disease. In this study, some patients started their medication with Taf-TFC. The treatment pattern of Taf-TFC was not significantly different for glaucoma type, age, baseline IOP, and baseline MD values. However, patients who started with Taf-TFC tended to have NTG, were 30-39 years old, had a baseline IOP of $25 \mathrm{mmHg}$ or higher, and had an MD value exceeding $-6 \mathrm{~dB}$. This information about "Naïve" patients receiving Taf-TFC monotherapy may be useful for physicians when selecting medication.

There were several limitations in this study. The major limitation of the study is the very short-term data. Unlike an interventional study, there was no control group, and specified examination and drug compliance could not be controlled in this observational study. Since we cannot define further the diagnosis of POAG and NTG, the SD of IOP reduction in this study was larger than in previous interventional studies, such as the phase III clinical trial of Taf-TFC [6]. Despite these limitations, these data reflect the actual real-world usage of Taf-TFC, and thus, our findings can serve as a reference for assessing the future use of Taf-TFC in clinical practice. Even though this study only analyzed short-term data up to 3 months, our results suggest that Taf-TFC is effective for the treatment of glaucoma in a variety of clinical settings. Since glaucoma usually requires chronic treatment, both long-term efficacy and safety data need to be collected and analyzed. This up to 2-year observational study is still ongoing, and the long-term findings will be presented after the data have been analyzed at the end of the study period. 


\section{CONCLUSION}

Evaluation of the short-term results obtained during 3 months after starting Taf-TFC treatment demonstrated that Taf-TFC is effective for a wide variety of glaucoma types or $\mathrm{OH}$ when administered in actual clinical practice with controllable or recoverable adverse reactions. Taf-TFC was effective regardless of regimens, and particularly, Taf-TFC significantly reduced IOP in patients insufficiently controlled on prior monotherapy.

\section{ACKNOWLEDGMENTS}

We wish to express our gratitude to the physicians at the medical institutions who participated in the study. Sponsorship and article processing charges for this study were funded by Santen Pharmaceutical Co., Ltd., Osaka, Japan. English language editing and manuscript preparation were performed by Yamada Translation Bureau, Inc and funded by Santen Pharmaceutical Co., Ltd (Osaka, Japan). All authors had full access to all of the data in this study and take complete responsibility for the integrity of the data and accuracy of the data analysis. All named authors meet the International Committee of Medical Journal Editors (ICMJE) criteria for authorship for this manuscript, take responsibility for the integrity of the work as a whole, and have given final approval for the version to be published.

Disclosures. Yasutaka Takagi, Hirotaka Osaki, Tomohiro Yamashita, and Yasuhiko Kai were employees of Santen Pharmaceutical Co., Ltd.

Compliance with Ethics Guidelines This current on-going post-marketing, multicenter, prospective, non-interventional, observational study is being conducted in accordance with the requirements of the regulatory authority in Japan and Good Post-Marketing Study Practice (MHLW Ordinance No. 171; December 20, 2004). The study began in January 2015 and is scheduled to run until September 2018. Since the protocol was reviewed and approved by the regulatory authority in Japan prior to beginning the study, approval by the ethics review committees of each participating medical institution was not required. The Japanese regulatory authority does not require informed consent for post-marketing observational studies, so informed consent was not obtained from the patients.

Open Access This article is distributed under the terms of the Creative Commons Attribution-NonCommercial 4.0 International License (http://creativecommons.org/licenses/ by-nc/4.0/), which permits any noncommercial use, distribution, and reproduction in any medium, provided you give appropriate credit to the original author(s) and the source, provide a link to the Creative Commons license, and indicate if changes were made.

\section{REFERENCES}

1. Schwartz GF, Quigley HA. Adherence and persistence with glaucoma therapy. Surv Ophthalmol. 2008;53:S57-68.

2. Juzych MS, Randhawa S, Shukairy A, et al. Functional health literacy in patients with glaucoma in urban settings. Arch Ophthalmol. $2008 ; 126: 718-24$.

3. Chen PP. Blindness in patients with treated open-angle glaucoma. Ophthalmology. 2003;110:726-33.

4. Marquis RE, Whitson JT. Management of glaucoma: focus on pharmacological therapy. Drugs Aging. $2005 ; 22: 1-21$. 
5. European Glaucoma Society. Terminology and guidelines for glaucoma. 4th ed. Savona: PubliComm; 2014.

6. Kuwayama Y, DE-111 Collaborative Trial Group. Phase III double-masked study of fixed combination tafluprost $0.0015 \% /$ timolol $0.5 \%$ (DE-111) versus tafluprost $0.0015 \%$ alone or given concomitantly with timolol $0.5 \%$ in primary open angle glaucoma and ocular hypertension. Atarasii Gannka (J Eye). 2013;30:1185-94 (in Japanese).

7. Kuwayama Y, DE-111 Collaborative Trial Group. Phase III double-masked study of fixed combination tafluprost $0.0015 \% /$ timolol $0.5 \%$ (DE-111) versus timolol $0.5 \%$ ophthalmic solution in primary open-angle glaucoma and ocular hypertension. Atarasii Gannka (J Eye). 2013;30:1773-81 (in Japanese).

8. Iwase A, Suzuki Y, Araie M, Yamamoto T, Abe H, Shirato $\mathrm{S}$, et al. The prevalence of primary open-angle glaucoma in Japanese: the Tajimi study. Ophthalmology. 2004;111:1641-8.

9. Yamamoto T, Iwase A, Araie M, Suzuki Y, Abe H, Shirato S, et al. The Tajimi study report 2: prevalence of primary angle closure and secondary glaucoma in a Japanese population. Ophthalmology. 2005;112:1661-9.

10. Yoshida A, Morimasa M, Bando E, Watanabe T, Komatsubara A, Endo Y. An interim report of the safety and efficacy of latanoprost and timolol maleate $\left(\right.$ Xalacom $^{\circledR}$ combination ophthalmic solution). Rinsho Ganka (Jpn J Clin Ophthalmol). 2014;68:573-80 in Japanese.

11. Ikeda Y, Mori K, Ishibashi T, Naruse S, Nakajima N, Kinoshita S. Latanoprost nonresponders with open-angle glaucoma in the Japanese population. Jpn J Ophthalmol. 2006;50:153-7.

12. Camras CB, Hedman K, US Latanoprost Study Group. Rate of response to latanoprost or timolol in patients with ocular hypertension or glaucoma. J Glaucoma. 2003;12:466-9.

13. Mizoguchi T, Ozaki M, Unoki K, Dake Y, Eto T, Arai M. A randomized crossover study comparing tafluprost $0.005 \%$ with travoprost $0.004 \%$ in patients with normal-tension glaucoma. Clin Ophthalmol. 2012;6:1579-84.
14. Gandolfi SA, Cimono L. Effect of bimatoprost on patients with primary open-angle glaucoma or ocular hypertension who are nonresponders to latanoprost. Ophthalmology. 2003;110:609-14.

15. Williams RD. Efficacy of bimatoprost in glaucoma and ocular hypertension unresponsive to latanoprost. Adv Ther. 2002;19:275-81.

16. Ueda K, Tonouchi A, Fukano Y, Asada H, Kawazu K. Tafluprost $\quad 0.0015 \% /$ timolol $0.5 \%$ combination ophthalmic solution (DE-111 ophthalmic solution) formulation design and intraocular penetration in rats. Atarashii Ganka (J Eye). 2013;30:1761-6 (in Japanese).

17. Heijl A, Leske MC, Bengtsson B, Hyman L, Bebgtsson B, Hussein $M$, et al. Reduction of intraocular pressure and glaucoma progression: results from the early manifest glaucoma trial. Arch Ophthalmol. 2002;120:1268-79.

18. Liu JH, Kripke DF, Weinreb RN. Comparison of the nocturnal effects of once-daily timolol and latanoprost on intraocular pressure. Am J Ophthalmol. 2004;138:389-95.

19. Takmaz T, Aşik S, Kürkçüoğlu P, Gurdal C, Can I. Comparison of intraocular pressure lowering effect of once daily morning vs evening dosing of latanoprost/timolol maleate combination. Eur J Ophthalmol. 2008;18:60-5.

20. Takeda N, Udagawa S, Higashide $\mathrm{T}$, Ohkubo $\mathrm{S}$, Takemoto Y, Sugiyama K. Comparison of intraocular pressure-reducing effect of morning vs. evening dosing of latanoprost and timolol. Atarasii Ganka (J Eye). 2016;33(3):455-9 (in Japanese).

21. Konstas AG, Tsironi S, Vakalis AN, Nasr MB, Stewart JA, Nelson LA, et al. Intraocular pressure control over 24 hours using travoprost and timolol fixed combination administered in the morning or evening in primary open-angle and exfoliative glaucoma. Acta Ophthalmol. 2009;87:71-6.

22. Djafari F, Lesk MR, Harasymowycz PJ, Desjardins D, Lachaine J. Determinants of adherence to glaucoma medical therapy in a long-term patient population. J Glaucoma. 2009;18:238-43. 